by bamorrhage from the mucous surfaces, as it is in small-pox, and yet without its being marked by any indisposition whatever, further than the slight feverishness which always follows, in the stage of areola, on the ninth and tenth days after the vaccination.

If the peculiarity had been occasioned by the Iymph introduced into practice some time ago by my friend, Mr. Ceely, of Aylesbury, by inoculating the cow with small-pox matter, and which lymph so obtained was for some time in use at this hospital, we might have supposed the case to be more nearly allied to variola than the vaccine disease arising spontaneously is generally allowed to be; and although I am no advocate, usually, or believer of the identity of the two, still I must say that this occurrence, so far as it goes, is in favour of their being of hin. dred origin. I have the honour to be, Sir, your obedient servant,

J. F. Marson, Surgeon,

Small-pox and Vaccination Hospital,

June 6, 1842.

\section{ARREST OF BLEEDING AFTER TOOTH-DRAWING.}

\section{To the Editor of The LANCET.}

SIR,-Upon perusing the paper of Dr. John Cochrane on hæomorrhagic diathesis in your invaluable Publication, the question arose in my mind, whether or not it had occurred to him, when treating the case last mentioned in his paper, to replace the tooth, as $I$ in one case replaced it upon the recommendation of a brother practitioner, and with perfect success.

In a case which occurred in my practice last week, a lady of midule age and weak fibre applied for some styptic to arrest the bleeding from the alveolus, after the extraction of the last molar by a dentist of some note. My assistant sent a powder consisting of alum, guin, and nut-galls, with which the socket was filled, but without success. I was then sent for, when I found the blood coming away of a florid hue, thin and watery; I then applied the compound tincture of Benjamin and a compress of lint, with no effect; afterwards the tincture of the sesquichloride of iron, which also failed; and the patient becoming faint, I gave her some brandy and water, when she rallied. I tried tincture of catechu and nitrate of silver with the same result; and, as Dr. Cochrane observes, the nitrate of silver arrested it for a short time, but not effectually, and eventually it was arrested with the spirit of turpentine.

The case, I think, proves the assertion of MI. Lloyd, of Liverpool, to be correct (that it is not dependent on age, sex, or condition of health, as she was and is in perfect health); and, also, it does not appear to be hereditary, as none of her family within her recollection ever suffered from hæmorrhage, being in this point opposed to Mr. Lane's opinion.

As the case has occurred so recently, and was illustrative of several points in Mr. Lloyd's opinion, and disproved that of $\mathrm{Mr}$. Lane, I have deemed it expedient to commit it to paper for a place in your Journal. I have the honour to be, Sir, your obedient servant,

$$
\begin{gathered}
\text { 52, Fore-sireet, Cripplegate, } \\
\text { May, 1842. }
\end{gathered}
$$

\section{QUESTIONS FROM DR. COOKSON.}

\section{To the Editor of The Lancet.}

Sir,-There is, as your pages show, only one way of silencing Mr. Hill, aud that consists in asking him a question which requires a straightforward answer, may I trouble you to put the following queries, and thus finish the dispute. Yours,

$$
\text { W. D. Cookson. }
$$

1. Where is any retraction of the deliberate statement made by $\mathbf{M r}$. K. to $\mathrm{Mr}$. Smith on the day of his discharge to be found?

2. Was the coroner put in possession of those suspicious circumstances respecting Mr. M.'s death, which were stated by Mr. $\mathrm{K}$. to $\mathrm{Mr}$. Smith, and afterwards repeated in writing to the board?

3. Was there not perforation of the windpipe ? and were there not marks of violence on the corpse?

4. Was Mr. Hill's resolution of the board unanimous?

5. Have not a large proportion of the governors present recorded their unequivocal dissent from the terms of that resolution?

6. Was not Mr. K.'s declaration, "that he had no complaint to make," an answer to a question put by the board respecting his Mi. K.'s own personal treatment?

7. Had the question the smallest reference to the treatment of others?

8. Did not Mr. K. expressly declare to Mr. Smith that his statement of the facts respecting Mr. M.'s death was not a complaint but a matter of conscience?

9. Will MIr. Hill venture to publish the whole transactions arising out of the case of $M r . M$. in the forthcoming report, the preparation of which, by an ingenious contrir. ance, has been placed in his hands?

** The above note was accidentally mislaid until a few days since. 